\title{
The Possible Risk of Reverse Zoonosis in COVID-19: An Epidemiological Driving Approach for the One Health Future Challenges: A Review
}

Viswanathan Naveenkumar, B.S. Pradeep $\mathrm{Nag}^{1}$, R. Vijayaraghavan, K. Porteen ${ }^{2}$

10.18805/ajdfr.DR-1543

\begin{abstract}
The emerging coronaviral infection named as COVID-19 was officially declared as pandemic on 11, March 2020 by WHO. It has so far been reported from 215 countries or territories affecting about twenty seven million people infected globally. The novel attributes on COVID-19 with sporadic reports on animal, alarms the future chances of animal mediated COVID-19 transmission. Despite lockdown in two-third of the global population, health officials are worried about the risky nature of animal infection in the current pandemic situation. The reverse zoonotic index cases in the current epidemic reported sporadically in animals through infected humans. Reported evidence suggests that bat as the major reservoir involved in COVID-19. However, still, the role of intermediate host involvement in the human COVID-19 transmission from the bat is not yet understood. It is clear that humans play a potent source of infection to transmit the disease to other humans and animals. A literature survey was conducted to a) understand the level of animal's involvement in COVID19 pandemic and b) to measure the amount of risk of reverse zoonoses in pet animals, exposed animals etc. The epidemiological investigation suggested the need for strong surveillance on the human-animal interface area with strict advisory measures to combat this dangerous disease transmission to humans and other animals. Hence understanding animal's role in the current pandemic is of prime importance in devising preparedness and control strategies through unique one health approach. In implementing suitable research protocol at animal-human interface along with environment by devising appropriate control strategies will reduce the future reverse zoonosis risk in the current pandemic through a holistic one health drive.
\end{abstract}

Key words: Animals, COVID-19, Host, One health, Reverse zoonosis.

The ongoing epidemics of severe acute respiratory syndrome coronavirus 2 (SARS-CoV 2) is one of the dangerous infectious diseases, so far reported in the millennium. At the fag end of the year 2019, Wuhan province, China reported a viral pneumonia infection whose origin was not known. Anamnesis revealed majority of the affected people was associated with local seafood animal market. Later, the virus was named SARS-CoV 2 due to its phenotypic as well as genotypic similarities with severe acute respiratory syndrome coronavirus (SARS-CoV) (Bedford et al., 2020; Heymann and Shindo, 2020; Wong et al., 2020). Severe contagiousness of SARS-CoV 2 has been attributed to the novel characteristics and poor knowledge about its epidemiological triad. Despite coronaviral lockdown in twothird of the world, researchers are continuously working to decipher SARS-CoV 2 virus epidemiology, transmission, host involved, therapeutics and vaccines. Reported evidences suggests that, bat is the principle host and also acts as a reservoir in the transmission of SARS-CoV 2 virus. However, the intermediate host and its role in the animal to human transmission is yet to be understood (Lake, 2020). The sequencing data suggests that bat and pangolin may be involved in the pandemic SARS-CoV 2 spread among human population (Guo et al., 2020; Lake, 2020; Zhang et al., 2020a and 2020b). Understanding the epidemiological triad which includes host, agent and environment during epidemics will play a pivotal role in devising effective control measures which eventually aims to curtail the epidemic
Department of Research and Development, Saveetha Institute of Medical and Technical Sciences, Chennai-602 105, Tamil Nadu, India.

${ }^{1}$ National Dairy Research Institute-SRS, Bengaluru-560 030, Karnataka, India.

2Department of Veterinary Public Health and Epidemiology, Madras Veterinary College, Tamil Nadu Veterinary and Animal Sciences University, Chennai-600 007, Tamil Nadu, India.

Corresponding Author: K. Porteen, Department of Veterinary Public Health and Epidemiology, Madras Veterinary College, Tamil Nadu Veterinary and Animal Sciences University, Chennai-600 007, Tamil Nadu, India. Email: rajavet2002@gmail.com

How to cite this article: Naveenkumar, V., Nag, B.S.P., Vijayaraghavan, R. and Porteen, K. (2020). The Possible Risk of Reverse Zoonosis in COVID-19: An Epidemiological Driving Approach for the One Health Future Challenges: A Review. Asian Journal of Dairy and Food Research. 39(3): 173-179.

Submitted: 13-06-2020 Accepted: 08-09-2020 Published: 17-09-2020

curve (Fig 1). This review aims to provide information regarding the role of animals in the current pandemic thorough literature survey and to discuss the level of risk in pet animals and exposed animals and issues related to one health approach in contending the current epidemics.

\section{History and Origin of COVID-19}

In December end of 2019, a group of people who had a 
The Possible Risk of Reverse Zoonosis in COVID-19: An Epidemiological Driving Approach for the One Health...

history of respiratory infection with unknown etiology was reported by Wuhan Municipal Health Commission, Wuhan, China. Most of the cases were closely associated with the local seafood market (Guo et al., 2020). The unknown pathogen was identified as a novel coronavirus ( $\mathrm{nCoV}$ ) on January 8, 2020, by Center for Disease Control and Prevention (CDC), China due to its genetic similarity and resemblance of clinical signs with the earlier reported coronaviral infections. Further, on $20^{\text {th }}$ of January human to human transmission of $\mathrm{nCoV}$ was officially reported. Meanwhile, Wuhan and other areas of China disturbed with huge epidemics of $\mathrm{nCoV}$ infection and showed a basic reproductive ratio (Ro) varied from 1.4 to 3.9 (Li et al., 2020). However, the transmission of $\mathrm{nCoV}$ crossed the national boundaries of China and reached Thailand on 13, January 2020 followed by several nations (Wu et al., 2020). Considering earlier data similarity with $\mathrm{nCoV}$, World Health Organisation (WHO) officially named as Coronavirus Diseases 2019 (COVID-19) and International Committee on Taxonomy of Viruses (ICTV) claimed it as severe acute respiratory syndrome coronavirus 2 (SARS-CoV 2) on February 11, 2020 (Du Toit, 2020; Gralinski and Menachery, 2020). World health organization declared the disease as pandemic considering its contagious load and severe spectrum of infection on March 11, 2020.

\section{Epidemiological association between animals and Coronaviridae family}

A coronavirus is a group of virus family that mainly infects respiratory and or enteric tract in multi-host species viz. avian, bat, masked palm civets, camels, mice, cattle, dogs, cats and humans (O'Connor et al., 2020). Coronavirus family consist of four genera viz., alpha, beta, gamma and delta coronaviral subtypes with many serotypes. The common mode of transmission is airborne droplets and or close contacts (Cavanagh, 2005).

In animals, commonly known coronaviral family diseases include transmissible gastroenteritis in pigs, bovine coronaviral infection in cattle, murine hepatitis virus in rat and mice, feline infectious peritonitis in cats and canine coronaviral infection in dogs (Cavanagh, 2005). In humans, so far seven coronaviral family diseases were reported and were mostly limited to the respiratory type of involvement (Chang et al., 2020). The categorical human coronaviral subtype mostly limited to beta coronavirus classification viz., HCoV-OC43, HCoV-HKU1, SARS-CoV, middle east respiratory syndrome coronavirus (MERS-CoV) and SARSCoV 2 and exceptionally in alphacoronaviral classification namely HCoV-229E and HCoV-NL63 (Cui et al., 2019; Hasoksuz et al., 2020).

The phylogenic evidence of SARS-CoV 2 claimed to be less similar with MERS-CoV (50-51.8\%) and comparatively higher association with SARS-CoV genome about to $79 \%$ similarity (Lu et al., 2020; Ren et al., 2020). Animals have been involved either as natural host and or intermediate host for all known human coronaviral diseases (Fig 2). This indicates, animal could play a potential role in transmission cycle of human corona virus epidemics (Cui et al., 2019). Additionally, the bat was the common natural host for the epidemics of HCoV-229E, HCoV-NL63, SARS-CoV, MERSCoV and SARS-CoV2. Similarly, in the case of HCoV-OC43 and HCoV-HKU1 epidemics, a rat was identified as a natural host. The intermediate host list for the human coronaviral diseases includes cattle, camel and civet cat (Cui et al., 2019; Hasoksuz et al., 2020). However, many of the researchers hypothesized the role of pangolin and or snakes as an intermediate host in the current COVID-19 epidemics (Zhang et al., 2020a and 2020b). Worldwide Pangolins with purported medicinal value are poached and trafficked for their meat and skin scales (Gao et al., 2020). Phylogenetic data analysis from field isolates of COVID-19, claimed pangolin as an intermediate host in the COVID-19 (Cui et al., 2019; Hasoksuz et al., 2020). However the precise role of pangolins in COVID-19 transmission cycle remains unclear till date.

\section{Experimental animal models for COVID-19}

The research community is continuously working to identify suitable animal model for the study of COVID-19 which would closely resemble the clinical manifestations expressed in humans in order to discover suitable diagnostic measures, therapeutics and vaccine. Retrospective data and the current research on human corona virus infections reveals that, the majority of human coronaviral diseases including COVID19 uses human angiotensin-conversion enzyme 2 (ACE2) as a doorstep to enter cell in the transmission chain (Jia et al., 2005; Demogines et al., 2012; Wan et al., 2020). So far experimental inoculation of COVID-19 in mice, monkey, ferret and Syrian hamster were studied in in silico and in vivo methodologies. None of the animal models has proved to simulate human clinical manifestations perfectly. However, intranasal inoculated ferret, Rhesus monkey and Syrian hamster showed similarities in expression of clinical symptoms, makes these animals a choice of study for COVID-19 (Callaway, 2020; Chan et al., 2020).

Transmission ability of the SARS-CoV 2 has been studied in intranasal inoculated animals experimentally which revealed that infected ferret and domestic cats affected the remaining ferrets and cats co-habitated with it, respectively (Halfmann et al., 2020; O'Connor et al., 2020). Similarly, studies were conducted to know the transmission ability in various livestock species viz. pigs, chicken and duck, suggested that transmission within the species was absent in spite of being housed with experimentally inoculated animals. However, no studies were conducted regarding cross species transmission.

\section{Reverse zoonosis of SARS-CoV and MERS-CoV from human to animal transmission}

An epidemiological surveillance study was conducted in the animal market of China and adjoining areas during the SARS-CoV outbreak from the livestock, wild and companion animal samples (Wang et al., 2005). In that report cat and wild boars were found to be positive and goats and dog 


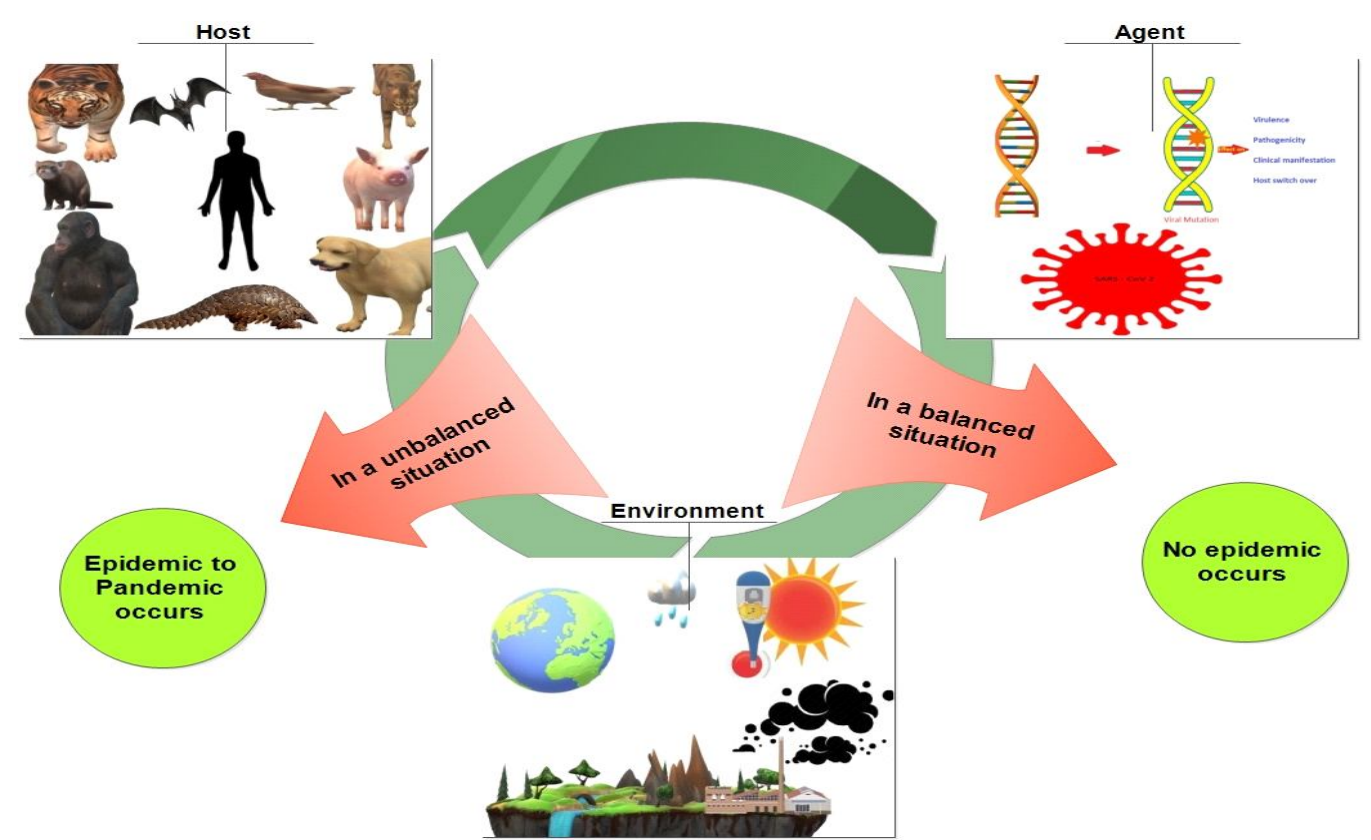

Fig 1: The epidemiological triad association in the current epidemic. In a balanced condition of $\mathrm{H}-\mathrm{A}-\mathrm{E}$, infectious disease epidemic would not occur whereas in unbalanced situation of $\mathrm{H}-\mathrm{A}-\mathrm{E}$ turn the no epidemic to epidemic and further pandemic is depicted.

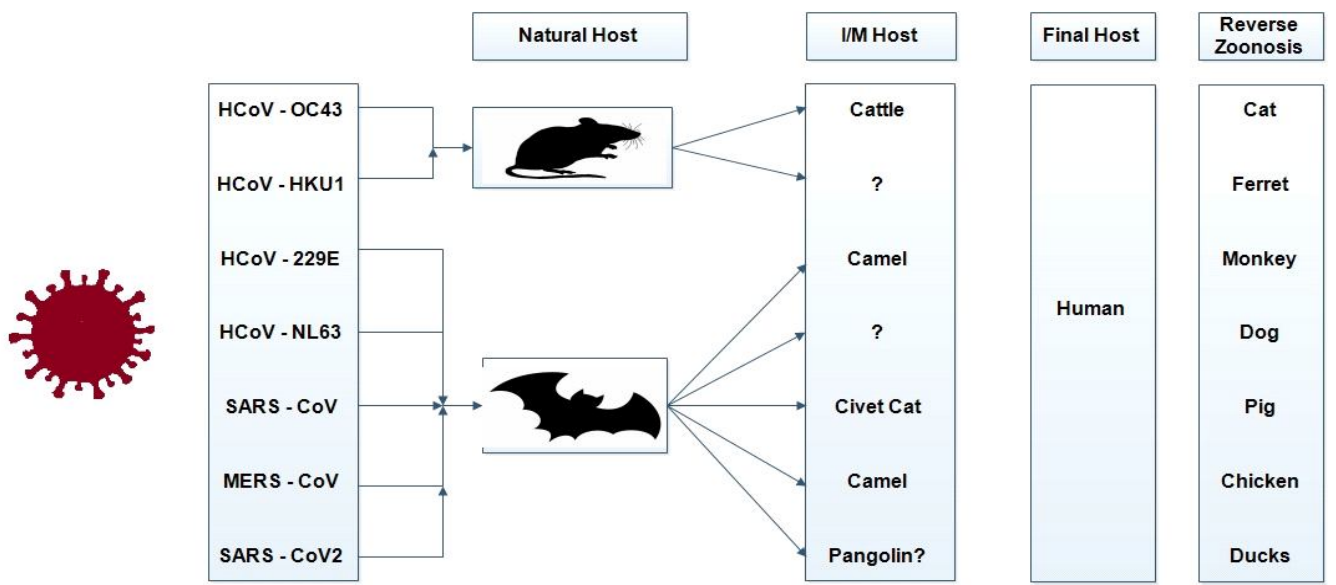

Fig 2: Animal host involvement in human coronaviral diseases. The known human coronaviral diseases along with their various host involvement were depicted. The phylogenetic association of Pangolin in the current epidemic was added. The chances of reverse zoonosis with the various animals list were reported in the SARS-CoV 2.

were negative for SARS-CoV through viral detection methods. Similarly, Chen et al. (2005) conducted a SARS field surveillance study on animals using antigen and antibody test methods and reported two positive cases of SARS in pigs for antibody detection. Of which, one pig showed positive for antibody, polymerase chain reaction (PCR) and virus isolation. Further sequence data strongly claimed that the transmission is from human origin SARSCoV. In their study dogs, cat and cattle were found to be negative for SARS.

Similarly, in MERS-CoV outbreak situations few reports of animal study were documented regarding the chances of animal infection. El-Duah et al. (2019) conducted a surveillance study on MERS-CoV from the field samples received from pig, sheep, goat, cattle and donkey of Ghana area and revealed that none of the samples were found positive for MERS. Kandeil et al. (2019) conducted surveillance study in Egypt, Tunisia and Senegal area samples from cattle, buffaloes, sheep, goat, donkey, mules and horses using antibody detection kit and PCR. The results revealed that both PCR and antibody tests were positive for MERS-CoV in sheep, goats, donkey and cattle species. However, PCR was negative for MERS-CoV in horses. Similarly, in Saudi Arabia, Kasem et al. (2018) conducted a study to diagnose MERS in sheep, goat and cattle samples which were closely associated with MERS affected human beings and found that, none of the samples were positive for MERS-CoV concluding that cross species transmission did not occurred. 
The Possible Risk of Reverse Zoonosis in COVID-19: An Epidemiological Driving Approach for the One Health...

Reported evidences suggests that SARS-CoV and MERS-CoV, viral transmissions occurred in human, domestic, wild and companion animals with a likelihood of human to animal transmission (Chen et al., 2005). However, still it is not clear whether infected animals shed the virus and are involved in transmission cycle. Though the cases were sporadic, compared to SARS-CoV and MERS-CoV epidemics, SARS-CoV 2 is considered to be a pandemic alert with huge epidemic overload in most of the countries. The risk assessment of earlier coronaviral incidence suggested that the possibility of host switch over and the pandemic nature of COVID-19 may express the huge chances of reverse zoonosis from humans to animals.

\section{Reverse zoonosis of COVID-19 from human to animal transmission}

To date, lockdown and social distancing are the topmost preventive measures followed by all over the world for people to reduce the further Ro value in COVID-19 pandemic. According to $\mathrm{WHO}$, social distancing is the major measure to be adopted by the people for a minimum of six months to a year. In this circumstance, pet animals are the major companion for the people to relieve lockdown stress (Vincent et al., 2020). Though the final host of COVID-19 is considered to be human, still the sporadic reports of human to animal transmission strengthen the chances of reverse zoonosis component from human to animal.

To date, there are few incidences of COVID-19 positive cases in animals that were reported sporadically viz., tiger (Bronx Zoo, New York), dog (Hong Kong) and cat (Belgium, Hong Kong and US). In Bronx Zoo, New York, a tiger named "Nadia" was reported with the ailment of dry cough and other respiratory illnesses. Further epidemiological investigation with diagnostic tests found to be positive for COVID-19 (Anonymous, 2020a; Parry, 2020). The hypothesis for the viral origin track was claimed to be from the asymptomatic zoo worker associated with the transmission of COVID-19 to the tiger. Despite positive reports, still, the shedding nature and infectivity, not yet reported for the other close contact animals.

In Hong Kong, two dog COVID-19 positive cases were reported from different pet household areas and who had a history of close association with their COVID-19 infected owners (O'Connor et al., 2020). Though the animals tested positive with PCR and antibody detection reports, there is no evidence of viral shedding and transmission to fellow animals housed with infected ones. The natural spill-over phenomenon might be a contributing factor for the positive evidence of COVID-19 in dogs. Goumenou et al. (2020) speculated that dogs could be the possible factor for the current epidemics load in northern Italy.

To date, three cat index cases (Belgium, Hong Kong and US) were identified as positive for COVID-19 and who had a history of close contact with diseased owners (Anonymous, 2020b; O'Connor et al., 2020). In a serosurveillance study from the cat population of Hong Kong with close contact from infected humans, revealed none of the samples was positive for COVID-19 (Zhang et al., 2020c). These findings were supported by Temmam et al. (2020), who reported zero positivity of COVID-19 from the 21 domestic pet animals with an associated history of diseased pet owners from France (12 dogs and 9 cats). IDEXX multinational veterinary biological company developed a new diagnostic kit for the COVID-19 and done surveillance study in many parts of the world with 4000 clinical samples from dogs, cats and horses with respiratory ailment. Though the animal samples were derived from COVID-19 prevalent area, none of the samples were positive for current pandemic disease (Anonymous, 2020c).

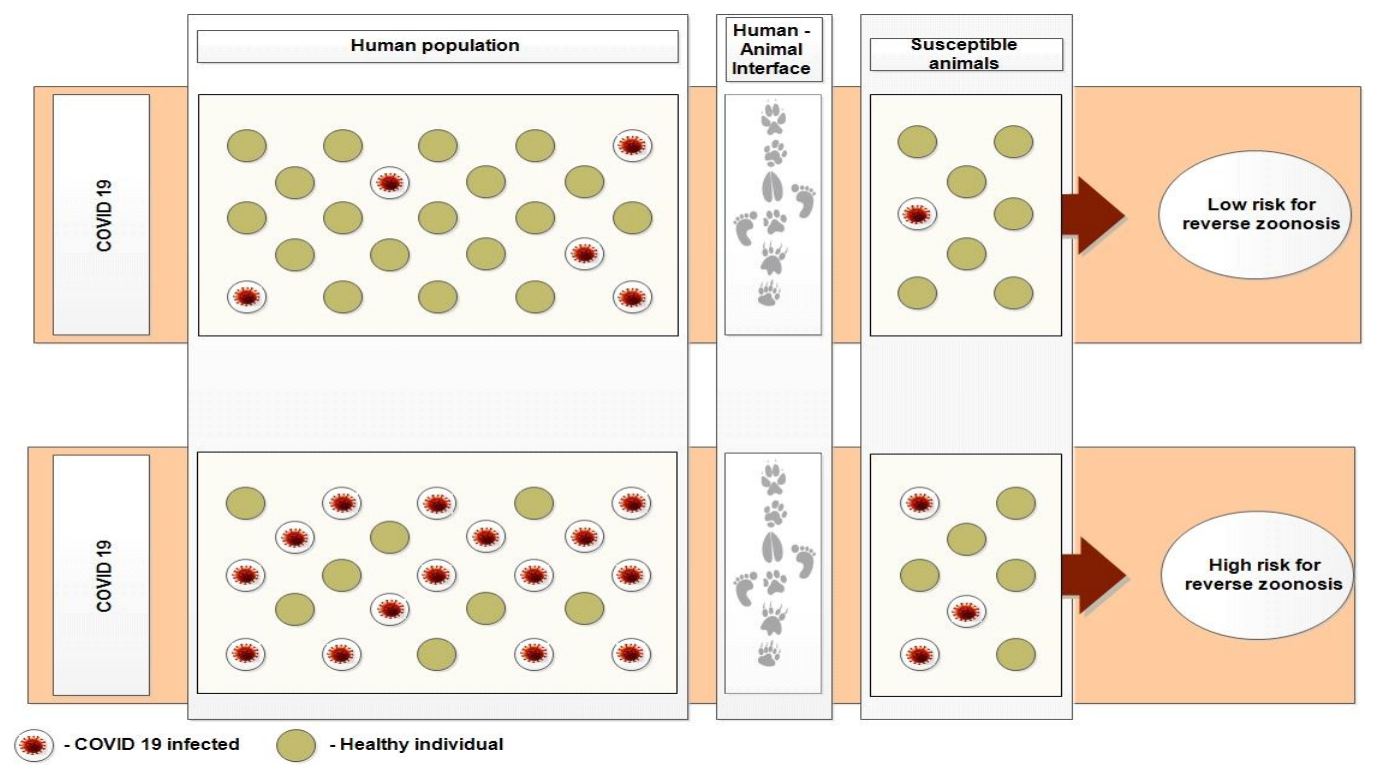

Fig 3: Risk profile of reverse zoonosis and the need of strong surveillance on COVID 19 at human animal interface area. The amount of infectivity in human population and the significance of human animal interface area depicted regarding risk nature of reverse zoonosis. 
The Possible Risk of Reverse Zoonosis in COVID-19: An Epidemiological Driving Approach for the One Health...

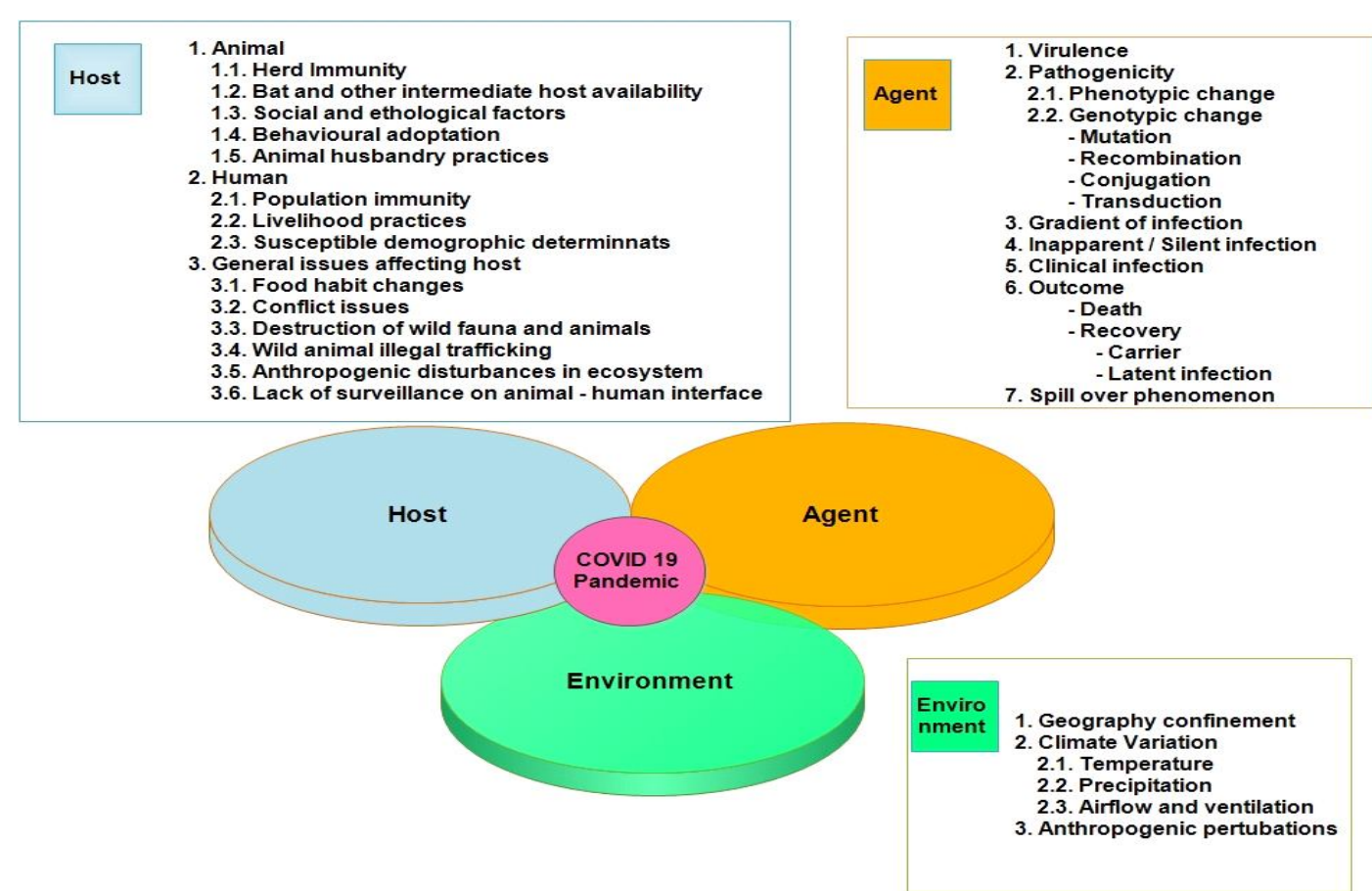

Fig 4: The role of host, agent and environment determinants in COVID 19. Various risk factors associated with current pandemic were reported with wide epidemiological approach.

Despite the sporadic reports of COVID-19 documented in few areas, still now the effective and strong evidence of reverse zoonosis is not yet accepted by $\mathrm{WHO}$ and it is postulated that, there is no evidence of pet animals mediated COVID-19 transmission to humans. However, CDC and all other health agencies addressed the need for strong surveillance study on COVID-19 especially in the area of human and animal interface (Fig 3). Few positive cases reported in pet animals, may not be oversized in the current pandemic and needs a strong look over animal health specially to protect animals from the infected population.

\section{Clinical dissimilarity of COVID-19}

Another phenomenon to worry about coronavirus pandemic is reinfection or relapse cases during the convalescence phase. In a longitudinal study reports published by Bao et al. (2020) ruled out relapse or reinfection of COVID-19 in Rhesus Monkey after cure. On the opposite side, Houser et al. (2017) conducted an experimental reinfection study of MERS-CoV in New Zealand rabbits and found that all the animals failed to develop a significant neutralizing antibody which makes the likelihood of getting reinfection. Though the similarity of MERS-CoV and SARS-CoV 2 is still unexplained, the majority of researchers claimed the reinfection risk is less in case of SARS-CoV 2.

The possible interlink information on host, agent and environment in the current pandemic were depicted in Fig 4. Among the earlier coronaviral infections identified, a canine enteric coronaviral vaccine was the only vaccines practiced all over the world as non-core vaccine regimen. Unfortunately, it would not give protection against COVID19 in pet animals due to the variation in the subtype of coronavirus involved for the respective disease (Anonymous, 2020d). Notwithstanding the fact, coronaviruses are having characteristics of genomic mutation which may affect the direction to find the vaccine candidate (Britton et al., 2020). Hence, adopting a vaccine for each disease is needed to combat this deadly viral family.

\section{CONCLUSION}

Even though, considerable amount of world population affected by COVID-19, still we are struggling to break the pandemic situation due to non-availability of vaccine and concrete treatment regimens. In a panic comparison, if pet animals or domestic animals association with COVID-19 may further affect the world disastrously. Lack of understanding in the clinical manifestations in animals limits our capability to diagnose the disease earlier, if reverses zoonosis occurs in this epidemic. This hypothesis may look to be threatened and in reality, it needs strong attention to build the "One Health" community in coming days. So far, the sporadic incidence may look weaker at this stage but might be a potent challenge for one health in future.

Based on existing reports of COVID-19 following measures may be recommended to check reverse zoonosis and minimize one health hazards in the current epidemics. The recommendations are; a). Infected persons should isolate themselves and surrounding animals, b). Before and after touching animals, proper washing and cleaning of hands with suitable disinfectant, c). Biohazards including a face 
The Possible Risk of Reverse Zoonosis in COVID-19: An Epidemiological Driving Approach for the One Health...

mask and personal protective equipment should be properly disposed according to the standard guidelines and d). Pets should not be entertained in endemic zones. Apart from these recommendations, some of the research protocol must be invocated in the human and animal interface area. They are; a). Periodic surveillance study of COVID-19 in animals especially should be conducted in possible human animal interaction zones and b). Random screening of pet animals which live in close contact with infected humans.

Exploration of teamwork between Medical and Veterinary Professionals is an inevitable approach to appraise and ascertain the probable risk factors involved in transmission between animals and humans. Such collaboration through unified ONE HEALTH approach will help to generate efficient scientific policies to control emergence of zoonotic diseases.

In a nutshell, sporadic reports of COVID-19 in animals suggests that the human is a potential source of infection to animal and is a possible risk element in the reverse zoonosis from human to animal spillover phenomenon. But still, it is unclear about the chance of carrier or source of infection from the infected animals to humans and or other animals. Hence, the concern regarding the reverse zoonosis in COVID-19 and further periodic screening of animals along with suggested advisory measures may help to combat future one health challenges in COVID-19 pandemic.

\section{Conflict of interest}

The authors declare that there is no conflict of interest regarding publication of this article.

\section{REFERENCES}

Anonymous. (2020a). United States Department of Agriculture, Animal and Plant Health Inspection Service. April 6, 2020. USDA Statement on the Confirmation of COVID-19 in a Tiger in NewYork, 2020. DOI: https://www.aphis.usda.gov/ aphis/newsroom/news/sa_by_date/ sa-2020/ny-zoocovid-19.

Anonymous. (2020b). Times of India, News. Two Cats in New York, Become First US Pets to Test Positive for Coronavirus. DOI: https://timesofindia.indiatimes.com/world/us/2cats-in-new-york-become-first-us-pets-to-test-positiveforcoronavirus/articleshow/75311029.cms.

Anonymous. (2020c). IDEXX March, IDEXX SARS-CoV-2 (COVID19) RealPCR Test. DOI: https://www.idexx.com/en/ veterinary/reference-laboratories/idexx-realpcr-tests/ idexx-sars-cov-2-covid-19-realpcr-test/.

Anonymous. (2020d). World Small Animal Veterinary Association. Advice for veterinarians about routine prophylactic vaccination during the COVID-19 pandemic. DOI: https:// wsava.org/news/highlighted-news/the-new-coronavirusand-companion-animals-advice-for-wsava-members/.

Bao, L., Deng, W., Gao, H., Xiao, C., Liu, J., Xue, J., Lv, Q., Liu, J., Yu, P., Xu, Y., Qi, F. (2020). Reinfection could not occur in SARS-CoV-2 infected rhesus macaques. bioRxiv. preprint. DOI: 10.1101/2020.03.13.990226.

Bedford, J., Enria, D., Giesecke, J., Heymann, D.L., Ihekweazu, C., Kobinger, G., Lane, H.C., Memish, Z., Oh, M.D.,
Schuchat, A., Ungchusak, K. (2020). COVID-19: towards controlling of a pandemic. The Lancet. 395(10229):10151018. DOI: 10.1016/S0140-6736(20)30673-5.

Britton, P., Armesto, M., Cavanagh, D., Keep, S. (2012). Modification of the avian coronavirus infectious bronchitis virus for vaccine development. Bioengineered. 3(2): 114-119. DOI: 10.4161 /bbug. 18983 .

Callaway, E. (2020). Labs rush to study coronavirus in transgenic animals-some are in short supply. Nature. 579(7798):183. DOI: 10.1038/d41586-020-00698-x.

Cavanagh, D. (2005). Coronaviridae: A Review of Coronaviruses and Toroviruses. In: Coronaviruses with Special Emphasis on First Insights Concerning SARS, Birkhäuser Basel. pp. 1-54. DOI: 10.1007/3-7643-7339-3_1.

Chan, J.F.W., Zhang, A.J., Yuan, S., Poon, V.K.M., Chan, C.C.S., Lee, A.C.Y., Chan, W.M., Fan, Z., Tsoi, H.W., Wen, L., Liang, R. (2020). Simulation of the clinical and pathological manifestations of Coronavirus Disease 2019 (COVID-19) in golden Syrian hamster model: implications for disease pathogenesis and transmissibility. Clinical Infectious Diseases. cia325. DOI: 10.1093/cid/ciaa325.

Chang, L., Yan, Y., Wang, L. (2020). Coronavirus disease 2019: coronaviruses and blood safety. Transfusion Medicine Reviews. 34: 75-80. DOI: 10.1016/j.tmrv.2020.02.003.

Chen, W., Yan, M., Yang, L., Ding, B., He, B., Wang, Y., Liu, X., Liu, C., Zhu, H., You, B., Huang, S. (2005). SARSassociated coronavirus transmitted from human to pig. Emerging Infectious Diseases. 11(3): 446-448. DOI: 10.3201/eid1103.040824.

Cui, J., Li, F., Shi, Z.L. (2019). Origin and evolution of pathogenic coronaviruses. Nature Reviews Microbiology. 17(3):181192. DOI: $10.1038 / \mathrm{s} 41579-018-0118-9$.

Demogines, A., Farzan, M., Sawyer, S.L. (2012). Evidence for ACE2utilizing coronaviruses (CoVs) related to severe acute respiratory syndrome CoV in bats. Journal of Virology. 86(11): 6350-6353. DOI: 10.1128/jvi.00311-12.

Du Toit, A. (2020). Outbreak of a novel coronavirus. Nature Reviews Microbiology. 18(3):123. DOI: 10.1038/s41579-020-0332-0.

El-Duah, P., Sylverken, A., Owusu, M., Yeboah, R., Lamptey, J., Oppong Frimpong, Y., Burimuah, V., Antwi, C., Folitse, R., Agbenyega, O., Oppong, S. (2019). Potential intermediate hosts for coronavirus transmission: No evidence of Clade 2c coronaviruses in domestic livestock from Ghana. Tropical Medicine and Infectious Disease. 4(1): 34. DOI: 10.3390/tropicalmed4010034.

Gao, W.H., Lin, X.D., Chen, Y.M., Xie, C.G., Tan, Z.Z., Zhou, J.J., Chen, S., Holmes, E.C., Zhang, Y.Z. (2020). Newly identified viral genomes in pangolins with fatal disease. Virus Evolution. 6(1): p.veaa020. DOI: 10.1093/ve/veaa020.

Goumenou, M., Spandidos, D.A., Tsatsakis, A. (2020). Possibility of transmission through dogs being a contributing factor to the extreme Covid 19 outbreak in North Italy. Molecular Medicine Reports. 21(6): 2293-2295. DOI: 10.3892/mmr. 2020.11037.

Gralinski, L.E. and Menachery, V.D. (2020). Return of the Coronavirus: 2019-nCoV. Viruses. 12(2):135. DOI: 10.3390/v12020135.

Guo, Y.R., Cao, Q.D., Hong, Z.S., Tan, Y.Y., Chen, S.D., Jin, H.J., Tan, K.S., Wang, D.Y., Yan, Y. (2020). The origin, trans-mission and clinical therapies on coronavirus disease 
The Possible Risk of Reverse Zoonosis in COVID-19: An Epidemiological Driving Approach for the One Health...

2019 (COVID-19) outbreak-an update on the status. Military Medical Research. 7(1): 1-10. DOI: 10.1186/ s40779-020-00240-0.

Halfmann, P.J., Hatta, M., Chiba, S., Maemura, T., Fan, S., Takeda, M., Kinoshita, N., Hattori, S.I., Sakai-Tagawa, Y., IwatsukiHorimoto, K., Imai, M. (2020). Transmission of SARSCoV-2 in Domestic Cats. New England Journal of Medicine. DOI: 10.1056/NEJMc2013400.

Hasoksuz, M., Kilic, S., Saraç, F. (2020). Coronaviruses and SARSCoV-2. Turkish Journal of Medical Sciences. 50(SI-1): 549-556. DOI: 10.3906/sag-2004-127.

Heymann, D.L. and Shindo, N. (2020). COVID-19: what is next for public health? The Lancet. 395: 542-545. DOI: 10.1016/ S0140-6736(20)30374-3.

Houser, K.V., Broadbent, A.J., Gretebeck, L., Vogel, L., Lamirande, E.W., Sutton, T., Bock, K.W., Minai, M., Orandle, M., Moore, I.N., Subbarao, K. (2017). Enhanced inflammation in New Zealand white rabbits when MERS-CoV reinfection occurs in the absence of neutralizing antibody. PLoS Pathogens. 13(8): p.e1006565. DOI: 10.1371/journal. ppat. 1006565.

Jia, H.P., Look, D.C., Shi, L., Hickey, M., Pewe, L., Netland, J., Farzan, M., Wohlford-Lenane, C., Perlman, S., McCray, P.B. (2005). ACE2 receptor expression and severe acute respiratory syndrome coronavirus infection depend on differentiation of human airway epithelia. Journal of Virology. 79(23): 14614-14621. DOI: 10.1128/jvi.79.23. 14614-14621.2005.

Kandeil, A., Gomaa, M., Shehata, M., El-Taweel, A., Kayed, A.E., Abiadh, A., Jrijer, J., Moatasim, Y., Kutkat, O., Bagato, O., Mahmoud, S. (2019). Middle East respiratory syndrome coronavirus infection in non-camelid domestic mammals. Emerging Microbes and Infections. 8(1): 103-108. DOI: 10.1080/22221751.2018.1560235.

Kasem, S., Qasim, I., Al-Hufofi, A., Hashim, O., Alkarar, A., AbuObeida, A., Gaafer, A., Elfadil, A., Zaki, A., Al-Romaihi, A., Babekr, N. (2018). Cross-sectional study of MERSCoV-specific RNA and antibodies in animals that have had contact with MERS patients in Saudi Arabia. Journal of Infection and Public Health. 11(3): 331-338. DOI: 10.1016/j.jiph.2017.09.022.

Lake, M.A. (2020). What we know so far: COVID-19 current clinical knowledge and research. Clinical Medicine. 20(2): 124127. DOI: 10.7861/clinmed.2019-coron.

Li, Q., Guan, X., Wu, P., Wang, X., Zhou, L., Tong, Y., Ren, R., Leung, K.S., Lau, E.H., Wong, J.Y., Xing, X. (2020). Early transmission dynamics in Wuhan, China, of novel coronavirus -infected pneumonia. New England Journal of Medicine. 382: 1199-1207. DOI: 10.1056/NEJMoa2001316.

Lu, R., Zhao, X., Li, J., Niu, P., Yang, B., Wu, H., Wang, W., Song, H., Huang, B., Zhu, N., Bi, Y. (2020). Genomic characterisation and epidemiology of 2019 novel coronavirus: implications for virus origins and receptor binding. The Lancet. 395: 565-574. DOI: 10.1016/S0140-6736 (20) 30251-8.

O'Connor, A.M., Totton, S.C., Sargeant, J.M. (2020). A rapid review of evidence of infection of pets and livestock with humanassociated coronavirus diseases, SARS, MERS and COVID-19 and evidence of the fomite potential of pets and livestock. Systematic reviews for animals and food, Available from: http://www.syreaf.org/wp-content/uploads/ 2020 /04/Rapid-Review-of-pets-as-fomites_3.pdf last accessed on 07-06-2020.

Parry, N.M. (2020). COVID-19 and pets: When pandemic meets panic. Forensic Science International: Reports. 2: 100090. DOI: 10.1016/j.fsir.2020.100090.

Ren, L.L., Wang, Y.M., Wu, Z.Q., Xiang, Z.C., Guo, L., Xu, T., Jiang, Y.Z., Xiong, Y., Li, Y.J., Li, X.W., Li, H. (2020). Identification of a novel coronavirus causing severe pneumonia in human: a descriptive study. Chinese Medical Journal. 133:1015-1024. DOI: 10.1097/CM9.0000000000000722.

Temmam, S., Barbarino, A., Maso, D., Behillil, S., Enouf, V., Huon, C., Jaraud, A., Chevallier, L., Backovic, M., Pérot, P., Verwaerde, P. (2020). Absence of SARS-CoV-2 infection in cats and dogs in close contact with a cluster of COVID19 patients in a veterinary campus. bioRxiv. preprint. DOI: 10.1101/2020.04.07.029090.

Vincent, A., Mamzer, H., Ng, Z., Farkas, K.J. (2020). People and their Pets in the Times of the COVID-19 Pandemic. Society Register. 4(3): 111-128.

Wan, Y., Shang, J., Graham, R., Baric, R.S., Li, F. (2020). Receptor recognition by the novel coronavirus from Wuhan: an analysis based on decade-long structural studies of SARS coronavirus. Journal of Virology. 94(7): e00127-20. DOI: 10.1128/jvi.00127-20.

Wang, M., Jing, H.Q., Xu, H.F., Jiang, X.G., Kan, B., Liu, Q.Y., Wan, K.L., Cui, B.Y., Zheng, H., Cui, Z.G., Yan, M.Y. (2005). Surveillance on severe acute respiratory syndrome associated coronavirus in animals at a live animal market of Guangzhou in 2004. Zhonghua liu xing bing xue za zhi= Zhonghua liuxingbingxue zazhi. 26(2): 84-87 (In Chinese). DOI: https://pubmed.ncbi.nlm.nih.gov/1592 $1605 /$.

Wong, G., Bi, Y.H., Wang, Q.H., Chen, X.W., Zhang, Z.G., Yao, Y.G. (2020). Zoonotic origins of human coronavirus 2019 (HCoV-19/SARS-CoV-2): why is this work important? Zoological Research. 41(3):1-7.

Wu, Y.C., Chen, C.S., Chan, Y.J. (2020). The outbreak of COVID19: An overview. Journal of the Chinese Medical Association. 83(3): 217-220. DOI: 10.1097/JCMA.000000000000 0270.

Zhang, Q., Zhang, H., Huang, K., Yang, Y., Hui, X., Gao, J., He, X., Li, C., Gong, W., Zhang, Y., Peng, C. (2020c). SARS-CoV-2 neutralizing serum antibodies in cats: a serological investigation. bioRxiv. preprint. DOI: 10.1101/2020.04.01.021196.

Zhang, Z., Wu, Q., Zhang, T. (2020a). Probable Pangolin Origin of SARS-CoV-2 Associated with the COVID-19 Outbreak. Current Biology. 30:1346-1351. DOI: 10.1016/j.cub. 2020. 03.022.

Zhang, Z., Wu, Q., Zhang, T. (2020b). Pangolin homology associated with 2019-nCoV. bioRxiv. preprint. DOI: 10.1101/2020.02. 19.950253. 